\title{
Editorial
}

\section{New Developments in Sliding Mode Control and Its Applications}

\author{
Ligang Wu, ${ }^{1}$ Rongni Yang, ${ }^{2}$ Guanghui Sun, ${ }^{1}$ Xudong Zhao, ${ }^{3}$ and Peng Shi,5 \\ ${ }^{1}$ Space Control and Inertial Technology Research Center, Harbin Institute of Technology, Harbin 150001, China \\ ${ }^{2}$ School of Control Science and Engineering, Shandong University, Jinan 250061, China \\ ${ }^{3}$ College of Information and Control Engineering, China University of Petroleum, Qingdao 266555, China \\ ${ }^{4}$ School of Electrical and Electronic Engineering, The University of Adelaide, Adelaide, SA 5005, Australia \\ ${ }^{5}$ College of Engineering and Science, Victoria University, Melbourne, VIC 8001, Australia \\ Correspondence should be addressed to Ligang Wu; ligangwu@hit.edu.cn
}

Received 15 June 2014; Accepted 15 June 2014; Published 22 July 2014

Copyright (C) 2014 Ligang Wu et al. This is an open access article distributed under the Creative Commons Attribution License, which permits unrestricted use, distribution, and reproduction in any medium, provided the original work is properly cited.

Since the invention of sliding mode control (SMC) in the early 1950s, the approaches to SMC algorithms and sliding mode estimation problems have been fully developed. SMC has developed into a general design method being examined for a wide spectrum of systems including nonlinear systems, uncertain systems, stochastic systems, and large-scale and infinite-dimensional systems. In spite of the extensive and successful development of SMC approaches and techniques, their capability to handle complex systems, high-order systems, singularly perturbed systems, real-life implementation, and chatter-free problem needs to be further strengthened. Meanwhile, newly developed mathematical tools and technologies have also opened up various possibilities for the advances in SMC. Until today, there are still many open problems in theoretical developments and practical applications of SMC.

In this special issue, most papers are concerned with the SMC applications; five papers consider the SMC problem for dynamical systems with time delays; three articles address SMC design for tracking systems; based on sliding mode observer, two articles focus on fault detection of networked control systems and parameter identification and synchronization of uncertain chaotic systems, respectively; there is also a single article introducing a novel fractional fast terminal SMC strategy for a class of dynamical systems with uncertainty; one article investigates a double power reaching law of SMC based on neural network; another paper discusses dynamic output-feedback control for a class of nonlinear systems with nonuniform uncertain sampling via TakagiSugeno (T-S) fuzzy control approach; finally, two articles cover SMC strategy for unbalanced load compensation and diesel engine air path subject to different disturbances.

During the past decades, the applications of SMC have received significant attention for different hybrid dynamical systems. "Sliding mode reference coordination of constrained feedback systems" by A. Vignoni et al. addresses the problem of coordinating dynamical systems with possibly different dynamics to achieve some desired collective behaviors under the constraints and capabilities of each system and "Sampleddata control of spacecraft rendezvous with discontinuous Lyapunov approach" by Z. Li et al. investigates the sampled-data stabilization problem of spacecraft relative positional holding with improved Lyapunov function approach. "Speed tracking and synchronization of a dual-motor system via second order sliding mode control" by W. Chen et al. is devoted to developing a new robust approach for the control of dual-motor systems by incorporating second order SMC techniques. "Second-order geometric sliding mode attitude observer with application to quadrotor on a test bench" by $\mathrm{H}$. An et al. is concerned with the sliding mode observer design framework and a second order geometric sliding mode attitude observer is designed for angular velocity estimation of quadrotor attitude. "Adaptive quasi-sliding mode control for permanent magnet DC motor" by F. E. Hoyos et al. solves the motor speed control problem of a buck power converter and DC motor coupled system by means of a quasi-sliding scheme and "Nonlinear control for trajectory tracking of a nonholonomic RC-hovercraft with discrete inputs" by D. Chaos et al. studies the problem of trajectory tracking for an underactuated RChovercraft by means of control with discrete inputs. " $L Q$ 
optimal sliding mode control of periodic review perishable inventories with transportation losses" by P. Leśniewski and A. Bartoszewicz applies the control-theoretic approach to design a new replenishment strategy for inventory systems with perishable stock. "Adaptive sliding mode robust control for virtual compound-axis servo system" by Y. Ren et al. proposes a structure mode of virtual compound-axis servo system to improve the tracking accuracy of the ordinary optoelectric tracking platform. "Sliding mode control for mass moment aerospace vehicles using dynamic inversion approach" by X.Y. Zhang et al. discusses modeling, control, and simulation of nonlinear six-degree-of-freedom mass moment aerospace vehicles based on fuzzy SMC and dynamic inversion techniques. "Sliding mode cooperative control for multirobot systems: a finite-time approach" by M. Ghasemi and S. G. Nersesov investigates a decentralized SMC technique, which guarantees finite-time coordination in multiagent systems, and "Fuzzy ARTMAP ensemble based decision making and application" by M. Jin et al. describes a new fuzzy ARTMAP ensemble approach based on the improved Bayesian belief method to improve the classification accuracy. "Barrier Lyapunov function-based sliding mode control for guaranteed tracking performance of robot manipulator" by S. Han et al. proposes a decentralized error-bounded SMC mechanism that ensures the prescribed tracking performance of a robot manipulator, while "Adaptive fuzzy dynamic surface sliding mode position control for a robot manipulator with friction and deadzone" by J. Y. Cheong shows a novel dynamic surface SMC scheme together with an adaptive fuzzy system, state observer, and parameter estimator to estimate the uncertainty, friction, and deadzone nonlinearities of a robot manipulator system. "Cooperative control of regenerative braking and antilock braking for a hybrid electric vehicle" by G. Yin and $\mathrm{X}$. Jin provides a new cooperative braking control strategy for a parallel hybrid electric vehicle with both a regenerative braking system and an antilock braking system to achieve improved braking performance and energy regeneration. "Adaptive backstepping finite time attitude control of reentry $R L V$ with input constraint" by F. Wang et al. solves the finitetime attitude tracking control problem of RLV under input constraint, model uncertainty, and external disturbance in reentry phase.

On the other hand, the SMC problem for dynamical systems with time delays has been investigated extensively, since delay is inherent in various engineering systems and is often a source of oscillation, instability, or poor performance of underlying control systems. "A novel approach to sliding mode control of time-delay systems" by $\mathrm{H}$. Xia et al. is concerned with the SMC for a class of linear systems with time-varying delay and establishes a sufficient condition to guarantee the asymptotical stability based on the delay fractioning approach. "Stability analysis of a class of second order sliding mode control including delay in input" by P. R. Acosta deals with the stability problems for a class of second order sliding mode systems. "Sliding mode control for multiagent system with time-delay and uncertainties: an LMI approach" by $\mathrm{P}$. Guo et al. considers the SMC of multiagent systems with time delay and uncertainties and a sufficient condition for time-delay independent sliding surface is obtained in LMI framework. "Observer-based sliding mode control for stabilization of a dynamic system with delayed output feedback" by B. Wang et al. constructs an observer based on delayed output feedback to realize the SMC control for stabilization of a dynamic delay system. "Robust estimation for discrete Markov system with time-varying delay and missing measurements" by J. You et al. addresses $H_{\infty}$ filtering problem for time-delayed Markov jump systems with intermittent measurements.

"Observer-based robust tracking control for a class of switched nonlinear cascade systems" by B. Niu et al. is devoted to robust output feedback tracking control design for a class of switched nonlinear cascade systems. "A chattering free discrete-time global sliding mode controller for optoelectronic tracking system" by Y. Ren et al. proposes a discrete-time global sliding mode controller for optoelectronic tracking system subject to uncertainties such as parameter variations and external disturbances. "A second-order sliding mode controller design for spacecraft tracking control" by Y.-X. Zhao et al. decomposes the spacecraft motion into threechannel subsystems, and a second-order SMC is proposed. "Fault detection of networked control systems based on sliding mode observer" by J. Zhang et al. is concerned with the network-based fault detection problem for a class of nonlinear discrete-time networked control systems with multiple communication delays and bounded disturbances, and a sliding mode based nonlinear discrete observer is proposed. "Parameter identification and synchronization of uncertain chaotic systems based on sliding mode observer" by L. Huang and L. Lin proposes a sliding mode state observer scheme that combines the SMC with observer theory and applies it to the uncertain chaotic system with unknown parameters and bounded interference.

"Fractional-order fast terminal sliding mode control for a class of dynamical systems" by G. Zhao introduces a novel fractional fast terminal SMC strategy for a class of dynamical systems with uncertainty. "A double power reaching law of sliding mode control based on neural network" by Y.-X. Zhao et al. develops an improved double power reaching law of SMC and a controller combined with neural network has been obtained. "Dynamic output-feedback passivity control for fuzzy systems under variable sampling" by $\mathrm{H}$. Li et al. concerns the problem of dynamic output-feedback control for a class of nonlinear systems with nonuniform uncertain sampling via T-S fuzzy control approach. On another active research front, "Integral sliding mode control strategy of D-STATCOM for unbalanced load compensation under various disturbances" by M. Xia and Y. Mao applies a two-loop control strategy based on the input-output feedback linearization and the integral sliding mode technique to D-STATCOM for unbalanced load compensation, while "Sliding mode control for diesel engine air path subject to matched and unmatched disturbances using extended state observer" by S. A. Ali and N. Langlois develops a sliding mode controller via a novel nonlinear disturbance observer for diesel engine air path system subject to matched and unmatched disturbances.

It is noted that SMC design for dynamic systems with time delays and tracking systems have always been hot issues in the field of control theory during the recent decades. On the other hand, many applications in engineering of SMC have also 
received considerable attention due to its broad practicality and significance in various areas. Recently, sliding mode observer scheme has attracted more and more research interests in different respects such as fault detection and parameter identification. As is well known, with increasingly largescale and complicated industrial processes, there are external disturbances originating from various sources in almost all controlled systems. Thus, the research of SMC strategy subject to different disturbances is a challenging problem. In summary, almost all articles in this special issue concern those most recent theoretical developments and practical applications of SMC.

Of course, this special issue is not a comprehensive representation of SMC. Nonetheless, it does represent the rich and many-faceted knowledge of the field and we hope the readers will find it well readable and useful as much as we do.

\section{Acknowledgments}

We would like to express our sincere appreciation to the authors for their remarkable contributions in assisting us. Finally, all reviewers' fundamental work on these papers is also very greatly acknowledged.

Ligang Wu

Rongni Yang

Guanghui Sun

Xudong Zhao

Peng Shi 


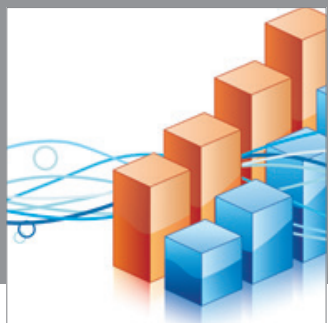

Advances in

Operations Research

mansans

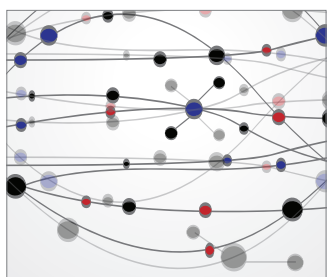

The Scientific World Journal
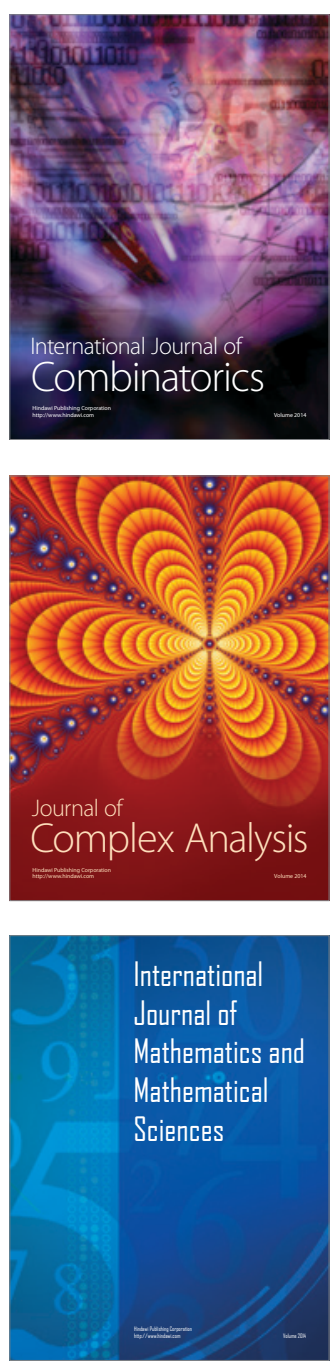
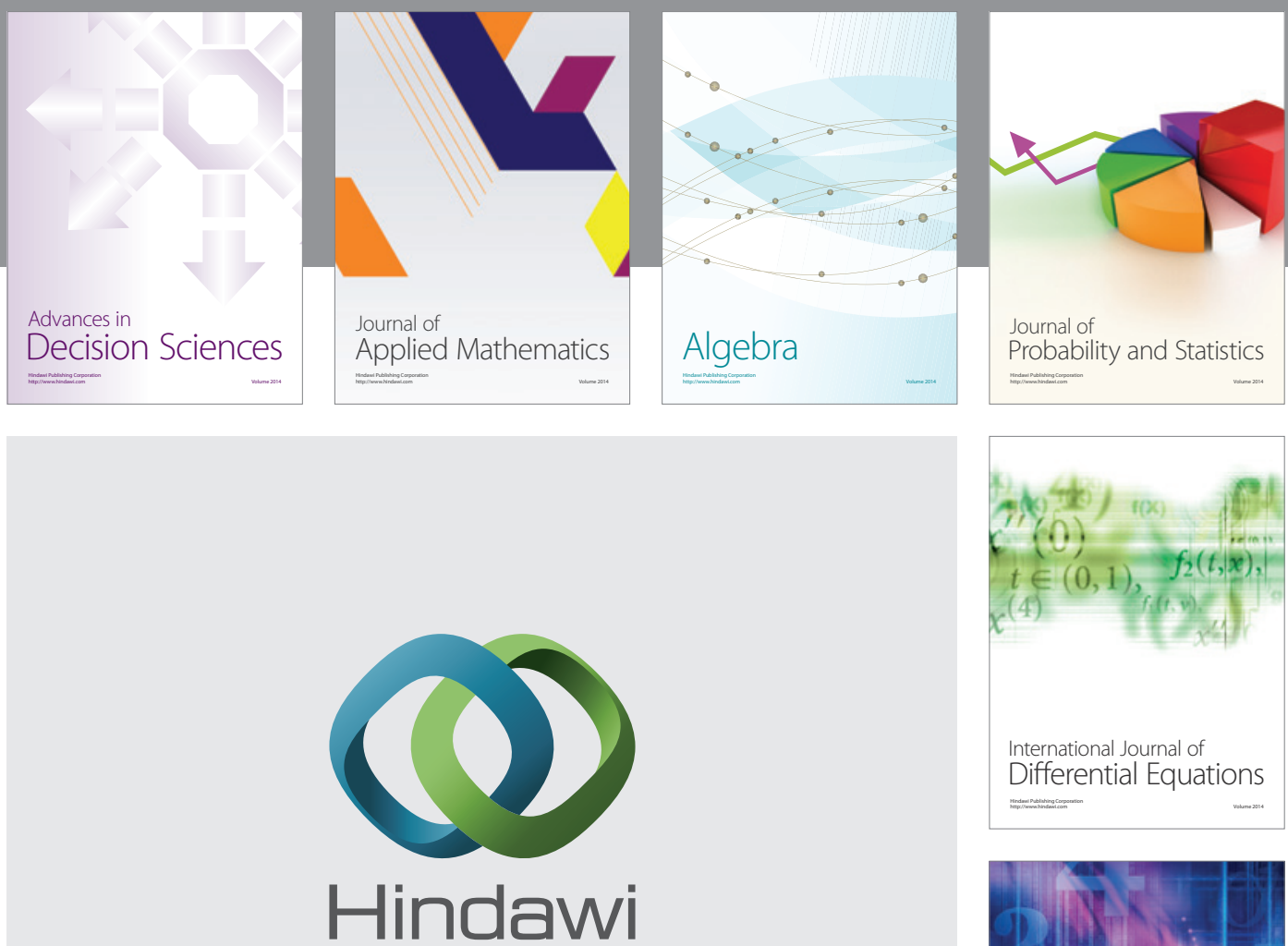

Submit your manuscripts at http://www.hindawi.com
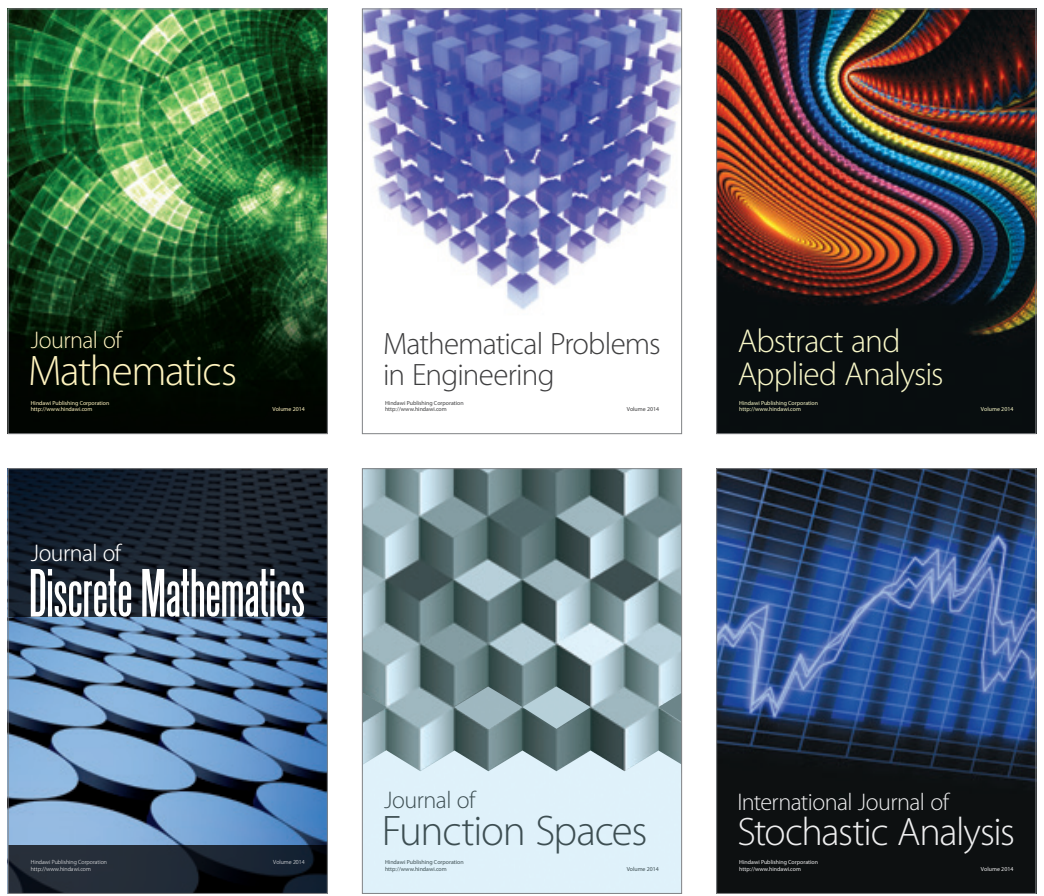

Journal of

Function Spaces

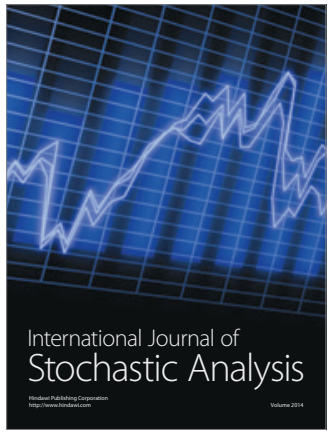

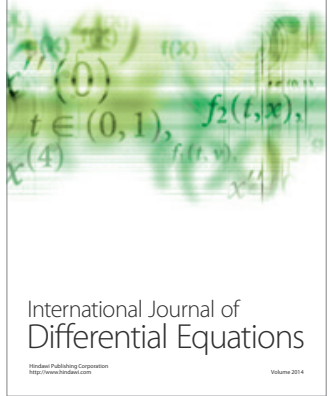
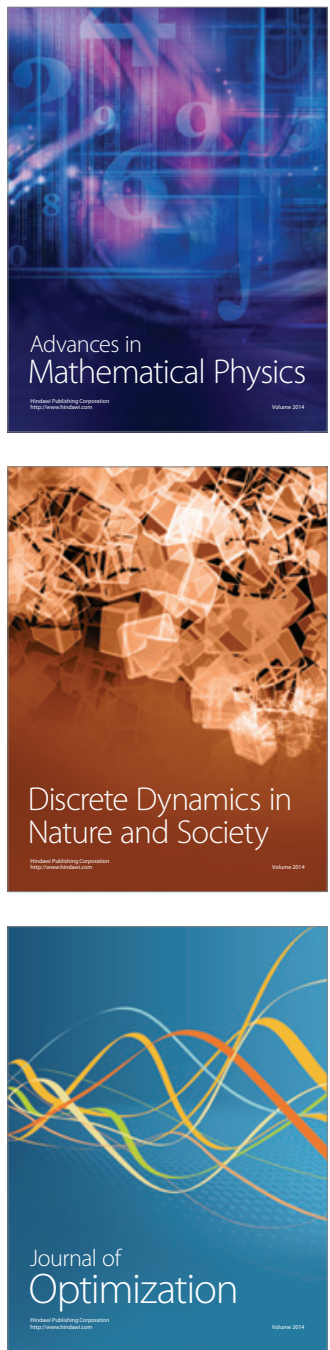